TRANSACTIONS OF THE

AMERICAN MATHEMATICAL SOCIETY

Volume 349, Number 4, April 1997, Pages 1447-1462

S 0002-9947(97)01504-3

\title{
INTEGER TRANSLATION OF MEROMORPHIC FUNCTIONS
}

\author{
JEONG H. KIM AND LEE A. RUBEL
}

\begin{abstract}
Let $G$ be a given open set in the complex plane. We prove that there is an entire function such that its integer translations forms a normal family in a neighborhood of $z$ exactly for $z$ in $G$ if and only if $G$ is periodic with period 1, i.e., $z \pm 1 \in G$ for all $z \in G$.
\end{abstract}

\section{INTRODUCTION AND PRELIMINARIES}

Let $\mathcal{S}$ be a space of functions defined on an open set $G$. For each positive integer $n$, we let $T_{n}$ be an operator from $\mathcal{S}$ into itself. Our concern is the normality of the family $\left\{T_{n}(f): n=0,1,2, \ldots\right\}$ for a function $f \in \mathcal{S}$. In this paper, we consider the case $T_{n}(f(z))=f(z+n)$, translation by $n$.

To discuss the normality of families of meromorphic functions, we need the following notion.

Definition 1.1. Let $f$ be a meromorphic function in the complex plane $\mathbf{C}$. We define the spherical derivative of $f$ at $z$ by

$$
\rho(f(z))=\frac{\left|f^{\prime}(z)\right|}{1+|f(z)|^{2}} .
$$

Theorem (Marty [6]). A family $\mathcal{F}$ of functions $f(z)$, meromorphic in a domain $D$, is normal in $D$ if and only if the set $\{\rho(f(z)): f \in \mathcal{F}\}$ is uniformly bounded on every compact subset of $D$.

Now we state our definition and an example.

Definition 1.2. For a meromorphic function $f$, we define the set $\mathbf{N}(f)$ to be the set of all $z_{0} \in \mathbf{C}$ such that the family $\{f(z+n): n=0,1,2, \ldots\}$ is normal in a neighborhood of $z_{0}$.

Example 1.3. Let $f(z)=z \sin \pi z$; then $\mathbf{N}(f)=\mathbf{C} \backslash \mathbf{Z}$ where $\mathbf{Z}$ is the set of all integers.

Proof. For all integers $n$,

$$
f(z+n)=(z+n) \sin \pi(z+n)=(z+n) \sin \pi z ;
$$

thus we have

$$
f(z+n) \begin{cases}=0, & \text { if } z \in \mathbf{Z}, \\ \rightarrow \infty, & \text { if } z \notin \mathbf{Z} \text { and } n \rightarrow \infty\end{cases}
$$

Received by the editors October 17, 1994 and, in revised form, March 31, 1995.

1991 Mathematics Subject Classification. Primary 30D45.

The research of the second author was partially supported by a grant from the National Science Foundation.

(C) 1997 American Mathematical Society 
Hence no subsequence of $\{f(z+n): n=0,1,2, \ldots\}$ converges on any neighborhood of any integer. But the sequence $\{f(z+n): n=0,1,2, \ldots\}$ converges uniformly to infinity on any compact subset which does not contain integers. These facts prove that $\mathbf{N}(f)=\mathbf{C} \backslash \mathbf{Z}$.

\section{Some properties of $\mathbf{N}(f)$}

From the definition, the set $\mathbf{N}(f)$ is open in the complex plane $\mathbf{C}$ and is periodic with period 1 .

The set $\mathbf{N}(f)$ may not be connected. For a certain meromorphic function $f$, the set $\mathbf{N}(f)$ has infinitely many components.

Example 2.1. Let $f(z)=\tan e^{z}$; then the set $\mathbf{N}(f)$ is not connected.

Proof. We let

$$
I_{c}=\{z \in \mathbf{C}: \operatorname{Im} z=k \pi, k=0, \pm 1, \pm 2, \ldots\},
$$

and we shall show that $\mathbf{N}(f)=\mathbf{C} \backslash I_{c}$. For $z=x+k \pi i \in I_{c}$, we have

$$
|f(z+n)|^{2}=\left|\tan e^{x+n+k \pi i}\right|^{2}=\tan ^{2} e^{x+n}
$$

and

$$
\left|f^{\prime}(z+n)\right|=\left|e^{x+n+k \pi i} \sec ^{2} e^{x+n+k \pi i}\right|=e^{x+n} \sec ^{2} e^{x+n} .
$$

So for every real number $x$ with $e^{x+n} \neq k \pi+\frac{\pi}{2}(k=0, \pm 1, \pm 2, \ldots)$,

$$
\begin{aligned}
\rho(f(z+n)) & =\frac{e^{x+n} \sec ^{2} e^{x+n}}{1+\tan ^{2} e^{x+n}} \\
& =\frac{e^{x+n}}{\sin ^{2} e^{x+n}+\cos ^{2} e^{x+n}} \\
& =e^{x+n} \rightarrow \infty
\end{aligned}
$$

as $n \rightarrow \infty$. But for an integer $l$ every neighborhood of $x+l \pi i$ contains a point $x^{\prime}+l \pi i$ such that $e^{x^{\prime}+n} \neq k \pi+\frac{\pi}{2}$. Hence by Marty's Theorem, $x+l \pi i \notin \mathbf{N}(f)$ for all real $x$.

Now for $z_{0} \notin I_{c}$, we choose a positive number $\varepsilon$ so that $B\left(z_{0}, \varepsilon\right) \cap I_{c}=\varnothing$. Let $F$ be a compact subset in $B\left(z_{0}, \varepsilon\right)$; then for $z=x+i y \in F$, we have

$$
\begin{aligned}
\rho(f(z+n)) & =\frac{\left|\left(\tan e^{z+n}\right)^{\prime}\right|}{1+\left|\tan e^{z+n}\right|^{2}} \\
& =\frac{\left|e^{z+n} \sec ^{2} e^{z+n}\right|}{1+\left|\tan e^{z+n}\right|^{2}} \\
& =\frac{\left|e^{z+n}\right|}{\left|\sin e^{z+n}\right|^{2}+\left|\cos e^{z+n}\right|^{2}} .
\end{aligned}
$$

For $z=x+i y$, it is easy to show

$$
|\sin z|^{2}+|\cos z|^{2}=\frac{1}{2}\left(e^{2 y}+e^{-2 y}\right)
$$

and since

$$
e^{z+n}=e^{x+n} \cos y+i e^{x+n} \sin y
$$

we can write

$$
\left|\sin e^{z+n}\right|^{2}+\left|\cos e^{z+n}\right|^{2}=\frac{1}{2}\left(e^{2 e^{x+n}} \sin y+e^{-2 e^{x+n} \sin y}\right) .
$$


Thus from (2.1),

$$
\begin{aligned}
\rho(f(z+n)) & =\frac{2 e^{x+n}}{e^{2 e^{x+n} \sin y}+e^{-2 e^{x+n} \sin y}} \\
& \leq \frac{2 e^{x+n}}{e^{2|\sin y| e^{x+n}}} .
\end{aligned}
$$

We let $\delta=\min \{|\sin y|: z=x+i y \in F\}$; then $\delta>0$. We define a function $g$ on the real line $\mathbf{R}$ by

$$
g(t)=\frac{2 e^{t}}{e^{2 \delta e^{t}}}
$$

then we get

$$
g^{\prime}(t)=2 e^{t-2 \delta e^{t}}\left(1-2 \delta e^{t}\right) .
$$

So the positive continuous function $g$ takes the maximum value $\frac{1}{\delta e}$ at $t=-\log 2 \delta$. Hence from (2.2), we have

$$
\rho(f(z+n)) \leq g(x+n) \leq \frac{1}{\delta e}
$$

for all $z \in F$ and $n=0,1,2, \ldots$. Therefore $z_{0}$ belongs to the set $\mathbf{N}(f)$. This completes the proof.

Let $\mathcal{A}$ be the space of all entire functions equipped with the topology of uniform convergence on compact subset of the complex plane $\mathbf{C}$.

Theorem 2.2. If $\mathcal{F}$ is a family of entire functions which is dense in $\mathcal{A}$, then the family $\mathcal{F}$ is not normal on any open set in $\mathbf{C}$.

Proof. For a given open set $\Omega$ and $z_{0} \in \Omega$, we choose a positive number $\varepsilon$ so that $\bar{B}\left(z_{0}, \varepsilon\right) \subset \Omega$ is a compact subset. By the density of $\mathcal{F}$, there exists a sequence $\left\{g_{n}(z)\right\} \subset \mathcal{F}$, such that

$$
\left|g_{n}(z)-n\left(z-z_{0}\right)\right|<\frac{1}{n}
$$

for all $z \in \bar{B}\left(z_{0}, \varepsilon\right)$. So we have

$$
\left|g_{n}\left(z_{0}\right)\right|<\frac{1}{n} \rightarrow 0
$$

as $n \rightarrow \infty$.

On the other hand, for $z \in \bar{B}\left(z_{0}, \varepsilon\right) \backslash\left\{z_{0}\right\}$,

$$
\left|g_{n}(z)\right|>n\left|z-z_{0}\right|-\frac{1}{n} \rightarrow \infty
$$

as $n \rightarrow \infty$. Hence the family $\mathcal{F}$ is not normal on $\Omega$.

An entire function $f$ whose translates are dense in $\mathcal{A}$ is called a universal entire function. In 1929, the existence of a universal entire function was proved by G. D. Birkhoff [1]. For a proof of the following theorem, see [5, pp. 60-61].

Theorem (Birkhoff [1]). There is an entire function whose integer translations are dense in the set $\mathcal{A}$ of all entire functions.

Birkhoff's Theorem and Theorem 2.2 have the following consequence.

Corollary 2.3. There is an entire function $f$ such that $\mathbf{N}(f)=\varnothing$. 
In Definition 1.2, we considered positive integer translations only. At this time we allow the negative integer translations also.

Definition 2.4. Let $f$ be a meromorphic function in the complex plane $\mathbf{C}$. We define as set $\mathbf{N}_{B}(f)$ by the set of all $z_{0} \in \mathbf{C}$ such that the family $\{f(z+n): n=$ $0, \pm 1, \pm 2, \ldots\}$ is normal at $z_{0}$.

By the same reasons as in $\mathbf{N}(f)$, the set $\mathbf{N}_{B}(f)$ is a periodic open set with period 1. And clearly $\mathbf{N}_{B}(f)$ is a subset of $\mathbf{N}(f)$. But $\mathbf{N}_{B}(f)$ is different from $\mathbf{N}(f)$ for a certain meromorphic function $f$.

Theorem 2.5. There is an entire function $f$ such that $\mathbf{N}_{B}(f) \neq \mathbf{N}(f)$.

Proof. We shall show that the entire function

$$
f(z)=\frac{e^{z^{2}}}{\Gamma(z)}
$$

satisfies the property by proving $0 \in \mathbf{N}(f) \backslash \mathbf{N}_{B}(f)$.

(i) $0 \notin \mathbf{N}_{B}(f)$ : Let $0<\varepsilon<\frac{1}{10}$ and $n$ be a positive integer. Since

$$
z \Gamma(z)=\Gamma(z+1)
$$

we have

$$
\begin{aligned}
\Gamma(\varepsilon-n) & =\frac{\Gamma(\varepsilon-n+1)}{\varepsilon-n} \\
& =\frac{\Gamma(\varepsilon)}{(\varepsilon-n)(\varepsilon-n+1) \cdots(\varepsilon-1)}
\end{aligned}
$$

and

$$
|f(\varepsilon-n)|=\left|\frac{e^{(\varepsilon-n)^{2}}(\varepsilon-n)(\varepsilon-n+1) \cdots(\varepsilon-1)}{\Gamma(\varepsilon)}\right| .
$$

The right-hand side of (2.3) tends to infinity as $n \rightarrow \infty$. But $f(-n)=0$ for all positive integers. Hence 0 does not belong to $\mathbf{N}_{B}(f)$.

(ii) $0 \in \mathbf{N}(f)$ : Since

$$
\frac{\Gamma^{\prime}(z)}{\Gamma(z)}=-\gamma+\sum_{m=1}^{\infty} \frac{z-1}{m(z+m-1)}
$$

where

$$
\begin{aligned}
\gamma & =\lim _{n \rightarrow \infty}\left(1+\frac{1}{2}+\frac{1}{3}+\cdots+\frac{1}{n}-\log n\right) \\
& =0.5772157 \cdots,
\end{aligned}
$$

we get

$$
\begin{aligned}
f^{\prime}(z) & =\frac{e^{z^{2}}}{\Gamma(z)}\left(2 z-\frac{\Gamma^{\prime}(z)}{\Gamma(z)}\right) \\
& =\frac{e^{z^{2}}}{\Gamma(z)}\left(2 z+\gamma-\sum_{m=1}^{\infty} \frac{z-1}{m(z+m-1)}\right) .
\end{aligned}
$$


Let $K$ be a compact subset of $B\left(0, \frac{1}{10}\right)$. Then for $z \in K$ and positive integer $n$,

$$
\begin{aligned}
\left|f^{\prime}(z+n)\right| & =\left|\frac{e^{(z+n)^{2}}}{\Gamma(z+n)}\left(2(z+n)+\gamma-\sum_{m=1}^{\infty} \frac{z+n-1}{m(z+n+m-1)}\right)\right| \\
& \leq\left|\frac{e^{(z+n)^{2}}}{\Gamma(z+n)}\right|\left(2(n+1)+\gamma+n \sum_{m=1}^{\infty} \frac{1}{m^{2}}\right) \\
& =\left|\frac{e^{(z+n)^{2}}}{\Gamma(z+n)}\right|\left(2(n+1)+\gamma+\frac{\pi^{2}}{6} n\right) .
\end{aligned}
$$

So we can write

$$
\begin{aligned}
\rho(f(z+n)) & \leq \frac{\left|\frac{e^{(z+n)^{2}}}{\Gamma(z+n)}\right|\left(2(n+1)+\gamma+\frac{\pi^{2}}{6} n\right)}{1+\left|\frac{e(z+n)^{2}}{\Gamma(z+n)}\right|^{2}} \\
& \leq\left|\frac{\Gamma(z+n)}{e^{(z+n)^{2}}}\right|\left(2(n+1)+\gamma+\frac{\pi^{2}}{6} n\right) .
\end{aligned}
$$

By Stirling's formula,

$$
\left|\frac{\Gamma(z+n)}{e^{(z+n)^{2}}}\right| \sim \frac{\left|\sqrt{2 \pi}(z+n)^{z+n-1 / 2} e^{-(z+n)}\right|}{\left|e^{(z+n)^{2}}\right|} .
$$

But for all $z \in K \subset B\left(0, \frac{1}{10}\right)$, we have

$$
\operatorname{Re}\left((z+n)^{2}+(z+n)\right) \geq n^{2}+\frac{n}{2}
$$

and

$$
|z+n|^{z+n-1 / 2}<e^{n \log (n+1)} .
$$

Thus we have

$$
\begin{aligned}
\frac{1}{n}- & \frac{\left|(z+n)^{z+n-1 / 2} e^{-(z+n)}\right|}{\left|e^{(z+n)^{2}}\right|} \\
& >\frac{1}{n}-\frac{e^{n \log (n+1)}}{e^{n^{2}+\frac{n}{2}}}=\frac{e^{n^{2}+\frac{n}{2}}-n e^{n \log (n+1)}}{n e^{n^{2}+\frac{n}{2}}} \\
& >\frac{e^{n^{2}}-e^{n \log (n+1)}}{e^{n^{2}+\frac{n}{2}}}>0 .
\end{aligned}
$$

With (2.5), for sufficiently large $n$ we obtain an inequality,

$$
\left|\frac{\Gamma(z+n)}{e^{(z+n)^{2}}}\right|<\frac{\sqrt{2 \pi}}{n} .
$$

So we can write $(2.4)$ as

$$
\rho(f(z+n)) \leq \frac{\sqrt{2 \pi}}{n}\left(2(n+1)+\gamma+\frac{\pi^{2}}{6} n\right)<16
$$

for all $z \in K$ and every positive integer $n$. Hence by Marty's theorem $0 \in \mathbf{N}(f)$. This completes the proof of the theorem. 


\section{MAin RESUlts}

Now we shall prove our main result. We know that, for every meromorphic function $f$, the set $\mathbf{N}(f)$ is a periodic open set with period 1 . To prove the existence part we shall construct an entire function with the help of the Weierstrass factorization theorem.

Theorem 3.1. Let $G$ be an open set in the complex plane $\mathbf{C}$. Then there exists an entire function $f$ such that $\mathbf{N}(f)=G$ if and only if $G$ is periodic with period 1 .

Theorem 3.1 is also true for the set $\mathbf{N}_{B}(f)$. We can prove it by a slight modification of the proof of Theorem 3.1.

Theorem 3.2. Let $G$ be an open set in the complex plane $\mathbf{C}$. Then there exists an entire function $f$ such that $\mathbf{N}_{B}(f)=G$ if and only if $G$ is periodic with period 1 .

Proof of Theorem 3.1. If $G=\mathbf{C}$, then we take a constant function for $f$. We assume $G$ is a proper subset of $\mathbf{C}$. Since $G$ is a periodic open set with period 1, it is enough to check for the set $\{z \in G: 0 \leq \operatorname{Re} z<1\}$ to show $\mathbf{N}(f)=G$. Because of its length we divide the proof into three steps and put each step as a section.

3.1. Construction of an entire function. Let $G$ be a periodic open set in the complex plane $\mathbf{C}$ with period 1 . For each positive integer $i$, we let

$$
W_{i}=\left\{w_{i j}: j \geq i\right\}
$$

be a countable dense subset of $R_{i}=\left\{z \in G^{c}: 0 \leq \operatorname{Re} z<1, i-1 \leq|\operatorname{Im} z|<i\right\}$ (for some $i$, the set $W_{i}$ can be empty or finite). We let $W=\bigcup_{i=1}^{\infty} W_{i}$ and

$$
G_{0}^{c}=\left\{z \in G^{c}: 0 \leq \operatorname{Re} z<1\right\} .
$$

Then $W$ is a countable dense subset of $G_{0}^{c}$.

From the set $W$, we form a sequence $Z=\left\{z_{i j}\right\}$ in $G^{c}$ as in Table 3.1 so that it has no finite limit point. In the construction of the sequence $Z$, for some positive

TABLE 3.1. Sequence $Z=\left\{z_{i j}\right\}$

\begin{tabular}{c|c|c|c}
\hline$C_{1}$ & $C_{2}$ & $C_{3}$ & $\cdots$ \\
\hline$z_{11}=w_{11}+2$ & $z_{12}=w_{11}+\left(2^{3}+1\right) !$ & $z_{13}=w_{11}+\left(3^{3}+1\right) !$ & $\cdots$ \\
& $z_{22}=w_{12}+\left(2^{3}+2\right) !$ & $z_{23}=w_{12}+\left(3^{3}+2\right) !$ & \\
& $z_{32}=w_{22}+\left(2^{3}+3\right) !$ & $z_{33}+=w_{22}+\left(3^{3}+3\right) !$ & \\
& & $z_{43}=w_{13}+\left(3^{3}+4\right) !$ & \\
& & $z_{53}=w_{23}+\left(3^{3}+5\right) !$ & \\
& & $z_{63}=w_{33}+\left(3^{3}+6\right) !$ & \\
\hline
\end{tabular}

integer $i$ if the set $W_{i}$ contains only finitely many elements, for instance, suppose that $W_{i}=\left\{w_{i j}: i \leq j \leq k\right\}$, and if $z_{s t}$ is a translation of $w_{i l}$ where $l>k$, then we skip the term $z_{s t}$. So if we let $\left\|C_{j}\right\|$ be the number of the elements in the $j$ th column of the Table 3.1, then we have

$$
\left\|C_{j}\right\| \leq \frac{j(j+1)}{2}
$$


And for each $z_{i j} \in C_{j}$, we have

$$
\operatorname{Re} z_{i j}<1+\left(j^{3}+\frac{j(j+1)}{2}\right) !
$$

and

$$
\left(j^{3}+1\right) ! \leq\left|z_{i j}\right|<j+1+\left(j^{3}+\frac{j(j+1)}{2}\right) !
$$

We define a function $g$ on the complex plane $\mathbf{C}$ by

$$
g(z)=\prod_{j=1}^{\infty} \prod_{z_{i j} \in C_{j}}\left(1-\frac{z}{z_{i j}}\right) .
$$

From (3.2) and (3.4) we have

$$
\begin{aligned}
\sum_{j=1}^{\infty} \sum_{z_{i j} \in C_{j}} \frac{1}{\left|z_{i j}\right|} & <\sum_{j=1}^{\infty} \frac{j(j+1)}{2} \frac{1}{\left(j^{3}+1\right) !} \\
& <e .
\end{aligned}
$$

Therefore the function $g$ is entire and its only zeros are at the points $z_{i j} \in Z$.

Finally, we define a function $f$ on the complex plane $\mathbf{C}$ by

$$
f(z)=e^{z} g(z) .
$$

Then $f$ is an entire function whose zero set is equal to that of $g$.

We shall show that $f$ is a suitable function.

3.2. For all $z \in G$, we have $z \in \mathbf{N}(f)$. For $z_{0} \in G$ (we assume $0 \leq \operatorname{Re} z_{0}<1$ ), $0<\varepsilon<\frac{1}{10}$, let $F=\bar{B}\left(z_{0}, \varepsilon\right)$ be a compact subset of $G$. Since $G^{c}$ is a closed set, there is a positive number $\varepsilon_{0}$ such that

$$
d\left(F, G^{c}\right)=\varepsilon_{0} .
$$

Let $n$ be a sufficiently large positive integer and suppose that $k$ is the largest integer such that

$$
1+\left(k^{3}+\frac{k(k+1)}{2}\right) !<n
$$

We fix $n$ for a moment and estimate the value of $|g(z+n)|$, for $z \in F$, and then we show $|f(n+z)| \rightarrow \infty$ uniformly on the compact set $F$ as $n \rightarrow \infty$.

To estimate the value $|g(z+n)|$, for $z \in F$, we consider the following four cases. Throughout this paper $C_{j}$ denotes the $j$ th column of Table 3.1.

Case 1. $j<k$

For each $z_{i j} \in C_{j}$ and $z \in F$, we have

$$
\begin{aligned}
\left|z_{i j}-(z+n)\right| & >\operatorname{Re}\left(z+n-z_{i j}\right) \\
& >n-2-\left(j^{3}+\frac{j(j+1)}{2}\right) !
\end{aligned}
$$


by (3.3). Thus from the inequalities (3.4) and (3.8), we obtain

$$
\begin{aligned}
\left|1-\frac{z+n}{z_{i j}}\right| & =\frac{1}{\left|z_{i j}\right|}\left|z_{i j}-(z+n)\right| \\
& >\frac{n-2-\left(j^{3}+\frac{j(j+1)}{2}\right) !}{j+1+\left(j^{3}+\frac{j(j+1)}{2}\right) !} \\
& >\frac{\left(k^{3}+\frac{k(k+1)}{2}\right) !-1-\left(j^{3}+\frac{j(j+1)}{2}\right) !}{j+1+\left(j^{3}+\frac{j(j+1)}{2}\right) !} \\
& >1 .
\end{aligned}
$$

Hence we can conclude

$$
\prod_{j=1}^{k-1} \prod_{z_{i j} \in C_{j}}\left|1-\frac{z+n}{z_{i j}}\right|>1
$$

Case 2. $j=k$

If $z_{i k}=w_{k k}+\left(k^{3}+\frac{k(k+1)}{2}\right) ! \in C_{k}$, then for all $z \in F$ we have

$$
\left|z_{i k}-(z+n)\right| \geq \varepsilon_{0}
$$

by (3.7). Thus by (3.4), we get

$$
\left|1-\frac{z+n}{z_{i k}}\right| \geq \frac{\varepsilon_{0}}{k+1+\left(k^{3}+\frac{k(k+1)}{2}\right) !} .
$$

Now we suppose $z_{i k} \neq w_{k k}+\left(k^{3}+\frac{k(k+1)}{2}\right)$ !. Then from Table 3.1, we get

$$
\operatorname{Re} z_{i k}<1+\left(k^{3}+\frac{k(k+1)}{2}-1\right) !
$$

and

$$
\left|z_{i k}\right|<k+1+\left(k^{3}+\frac{k(k+1)}{2}-1\right) !
$$

Hence for each $z_{i k} \in C_{k}\left(z_{i k} \neq w_{k k}+\left(k^{3}+\frac{k(k+1)}{2}\right) !\right)$, we have

$$
\begin{aligned}
\left|1-\frac{z+n}{z_{i k}}\right| & =\frac{1}{\left|z_{i k}\right|}\left|z_{i k}-(z+n)\right| \\
& \geq \frac{n-\operatorname{Re} z_{i k}-1}{\left|z_{i k}\right|} \\
& \geq \frac{n-2-\left(k^{3}+\frac{k(k+1)}{2}-1\right) !}{k+1+\left(k^{3}+\frac{k(k+1)}{2}-1\right) !}
\end{aligned}
$$

But we defined $k \geq 2$ in (3.8) so that $1+\left(k^{3}+\frac{k(k+1)}{2}\right) !<n$. Thus

$$
\begin{aligned}
\left|1-\frac{z+n}{z_{i k}}\right| & >\frac{\left(k^{3}+\frac{k(k+1)}{2}\right) !-\left(k^{3}+\frac{k(k+1)}{2}-1\right) !-1}{k+1+\left(k^{3}+\frac{k(k+1)}{2}-1\right) !} \\
& >1 .
\end{aligned}
$$

From (3.10) and (3.11), we conclude 


$$
\prod_{z_{i k} \in C_{k}}\left|1-\frac{z+n}{z_{i k}}\right|>\frac{\varepsilon_{0}}{k+1+\left(k^{3}+\frac{k(k+1)}{2}\right) !}
$$

for all $z \in F$.

Case 3. $j=k+1$

We shall show that

$$
\prod_{x_{i j} \in C_{k+1}}\left|1-\frac{z+n}{z_{i j}}\right|>\varepsilon_{0}^{2} \frac{\left[\left((k+1)^{3}+2\right) !-\left((k+1)^{3}+1\right) !\right]^{\frac{(k+1)(k+2)}{2}-2}}{\left[k+2+\left((k+1)^{3}+\frac{(k+1)(k+2)}{2}\right) !\right]^{\frac{(k+1)(k+2)}{2}}}
$$

for all $z \in F$.

Let $z_{\alpha j}, z_{\beta_{j}} \in C_{j}$ be the first and second nearest points to the compact set $F+n$; then from (3.7) and Table 3.1, we have

$$
\left|z_{i j}-(z+n)\right| \geq \begin{cases}\varepsilon_{0}, & \text { if } z_{i j}=z_{\alpha j} \text { or } z_{\beta_{j}}, \\ \left(j^{3}+2\right) !-\left(j^{3}+1\right) !, & \text { if } z_{i j} \neq z_{\alpha j} \text { and } z_{\beta_{j}} .\end{cases}
$$

So by (3.2) and (3.4), we have

$$
\begin{aligned}
\prod_{z_{i j} \in C_{j}}\left|1-\frac{z+n}{z_{i j}}\right| & \geq \frac{\prod_{z_{i j} \in C_{j}}\left|z_{i j}-(z+n)\right|}{\left(j+1+\left(j^{3}+\frac{j(j+1)}{2}\right) !\right)^{\frac{j(j+1)}{2}}} \\
& >\varepsilon_{0}^{2} \frac{\left(\left(j^{3}+2\right) !-\left(j^{3}+1\right) !\right)^{\frac{j(j+1)}{2}-2}}{\left(j+1+\left(j^{3}+\frac{j(j+1)}{2}\right) !\right)^{\frac{j(j+1)}{2}}} .
\end{aligned}
$$

Put $j=k+1$ into (3.14); then it becomes (3.13).

Case 4. $j>k+1$

We define a sequence $\left\{\alpha_{k}\right\}$ by

$$
a_{k}=\min _{\substack{z \in F \\ n \in N(k)}}\left|\prod_{j=k+2}^{\infty} \prod_{z_{i j} \in C_{j}}\left(1-\frac{z+n}{z_{i j}}\right)\right|
$$

where

$$
N(k)=\left\{n: 1+\left(k^{3}+\frac{k(k+1)}{2}\right) !<n \leq 1+\left((k+1)^{3}+\frac{(k+1)(k+2)}{2}\right) !\right\} .
$$

Since $F \subset G$, by the periodicity of $G$, we have $F+n \subset G$ for all positive integers $n$. Hence $|g(z+n)| \neq 0$ for all $z \in F$. Thus $a_{k}>0$ for all positive integers $k$. 
We shall show that $\left\{a_{k}\right\}$ is eventually an increasing sequence. For convenience, we write $\min _{z, n_{1}}$ and $\min _{z^{\prime}, n_{2}}$ instead of $\min _{n_{1} \in N(k)}^{z \in F}$ and min $\underset{n_{2} \in N(k+1)}{z^{\prime} \in F}$ (and likewise for max). To prove that $a_{k}$ is increasing, we examine the ratio:

$$
\begin{aligned}
\frac{a_{k+1}}{a_{k}} \geq & \frac{\min _{z^{\prime}, n_{2}} \mid \prod_{j=k+3}^{\infty} \prod_{z_{i j}^{\prime} \in C_{j}}\left(1-\frac{z^{\prime}+n_{2}}{z_{i j}^{\prime}} \mid\right.}{\max _{z^{\prime}, n_{1}} \mid \prod_{j=k+2}^{\infty} \prod_{z_{i j} \in C_{j}}\left(1-\frac{z+n_{1}}{z_{i_{j}}^{\prime}} \mid\right.} \\
\geq & \min _{z, n_{1}} \prod_{z_{i j} \in C_{k+2}}\left|\frac{z_{i j}}{z_{i j}-\left(z+n_{1}\right)}\right| \times \frac{\min _{z^{\prime}, n_{2}} \prod_{z_{i j}^{\prime} \in C_{k+3}}\left|\frac{z_{i j}^{\prime}-\left(z^{\prime}+n_{2}\right)}{z_{i j}^{\prime}}\right|}{\max _{z, n_{1}} \prod_{z_{i j} \in C_{k+3}}\left|\frac{z_{i j}-\left(z+n_{1}\right)}{z_{i j}}\right|} \\
& \times \frac{\min _{z^{\prime}, n_{2}} \prod_{z_{i j}^{\prime} \in C_{k+4}}\left|\frac{z_{i j}^{\prime}-\left(z^{\prime}+n_{2}\right)}{x_{i j}^{\prime}}\right|}{\max _{z, n_{1}} \prod_{z_{i j} \in C_{k+4}}\left|\frac{z_{i j}-\left(z+n_{1}\right)}{z_{i j}}\right|} \times \cdots \\
\geq & \min _{z, n_{1}} \prod_{z_{i j} \in C_{k+2}}\left|\frac{z_{i j}}{z_{i j}-\left(z+n_{1}\right)}\right| \times \frac{\min _{z^{\prime}, n_{2}} \prod_{z_{i j}^{\prime} \in C_{k+3}}\left|z_{i j}^{\prime}-\left(z^{\prime}+n_{2}\right)\right|}{\max _{z, n_{1}} \prod_{z_{i j} \in C_{k+3}}\left|z_{i j}-\left(z+n_{1}\right)\right|} \\
\quad & \frac{\min _{z^{\prime}, n_{2}} \prod_{z_{i j}^{\prime} \in C_{k+4}}\left|z_{i j}^{\prime}-\left(z^{\prime}+n_{2}\right)\right|}{\max _{z, n_{1}} \prod_{z_{i j} \in C_{k+4}}\left|z_{i j}-\left(z+n_{1}\right)\right|} \times \cdots \\
\quad & \min _{z, n_{1}} \prod_{z_{i j} \in C_{k+2}}\left|\frac{z_{i j}}{z_{i j}-\left(z+n_{1}\right)}\right| \times \min _{z^{\prime}, n_{2}} \prod_{z_{i j}^{\prime} \in C_{k+3}}\left|z_{i j}^{\prime}-\left(z^{\prime}+n_{2}\right)\right| \\
& \times \frac{\min _{z^{\prime}, n_{2}} \prod_{z_{i j}^{\prime} \in C_{k+4}}\left|z_{i j}^{\prime}-\left(z^{\prime}+n_{2}\right)\right|}{\max _{z, n_{1}} \prod_{z_{i j} \in C_{k+3}}\left|z_{i j}-\left(z+n_{1}\right)\right|} \\
& \times \frac{\min _{z^{\prime}, n_{2}} \prod_{z_{i j}^{\prime} \in C_{k+5}}\left|z_{i j}^{\prime}-\left(z^{\prime}+n_{2}\right)\right|}{\max _{z, n_{1}} \prod_{z_{i j} \in C_{k+4}}\left|z_{i j}-\left(z+n_{1}\right)\right|} \times \cdots .
\end{aligned}
$$

We can easily check that the first and second terms of the last equation of (3.16) are greater than 1. From (3.4) and Table 3.1, for all $z \in F, n_{1} \in N(k)$ and $z_{i j} \in C_{k+l-1}$ $(l \geq 4)$, we have

$$
\left|z_{i j}-\left(z+n_{1}\right)\right| \leq\left|z_{i j}\right| \leq k+l+\left((k+l-1)^{3}+\frac{(k+l-1)(k+l)}{2}\right) !
$$

and for $z^{\prime} \in F, n_{2} \in N(k+1)$ and $z_{i j}^{\prime} \in C_{k+l}(l \geq 4)$, we have

$$
\begin{aligned}
\left|z_{i j}^{\prime}-\left(z+n_{2}\right)\right| & \geq \operatorname{Re}\left|z_{i j}^{\prime}-\left(z+n_{2}\right)\right| \\
& \geq\left((k+l)^{3}+1\right) !-2-\left[\left((k+2)^{3}+\frac{(k+2)(k+3)}{2}\right) !\right] \\
& >\left((k+l)^{3}+1\right) !-(k+3)^{3} ! .
\end{aligned}
$$

So we have an inequality, 


$$
\begin{aligned}
\mid z_{i j}^{\prime}- & \left(z^{\prime}+n_{2}\right)|-| z_{i j}-\left(z+n_{1}\right) \mid \\
> & \left((k+l)^{3}+1\right) !-(k+3)^{3} ! \\
& -\left[k+l+\left((k+l-1)^{3}+\frac{(k+l-1)(k+l)}{2}\right) !\right] \\
= & (k+3)^{3} !\left[(k+l)^{3}+1\right) \cdots\left((k+3)^{3}+1\right)-1 \\
& \left.-\left(k+l+(k+l-1)^{3}+\frac{(k+l-1)(k+l)}{2}\right) \cdots\left((k+3)^{3}+1\right)\right] .
\end{aligned}
$$

It is easy to check that

$$
(k+l)^{3}+1>k+l+(k+l-1)^{3}+\frac{(k+l-1)(k+l)}{2}
$$

for $l \geq 4$. Hence the right-hand side of (3.17) is greater than 0 and we have

$$
\left|\frac{z_{i j}^{\prime}-\left(z^{\prime}+n_{2}\right)}{z_{i j}-\left(z+n_{1}\right)}\right|>1
$$

for all $z, z^{\prime} \in F, n_{1} \in N(k), n_{2} \in N(k+1), z_{i j} \in C_{k+l-1}$ and $z_{i j}^{\prime} \in C_{k+l}$. Since $\left\|C_{k+l}\right\| \geq\left\|C_{k+l-1}\right\|$, we have

$$
\frac{\min _{z^{\prime}, n_{2}} \prod_{z_{i j}^{\prime} \in C_{k+l}}\left|z_{i j}^{\prime}-\left(z^{\prime}+n_{2}\right)\right|}{\max _{z, n_{1}} \prod_{z_{i j} \in C_{k+l-1}}\left|z_{i j}-\left(z+n_{1}\right)\right|}>1
$$

for all $l \geq 4$.

Now we can conclude that $\frac{a_{k+1}}{a_{k}}>1$. Therefore $\left\{a_{k}\right\}$ is a positive and eventually increasing sequence.

From (3.9), (3.12), (3.13) and (3.15), for all $z \in f$ and $n \in N(k)$, we can write

$$
\begin{aligned}
|f(z+n)|= & \left|e^{z+n} g(z+n)\right| \\
= & \left|e^{z+n}\right| \times \prod_{j=1}^{k-1} \prod_{z_{i j} \in C_{j}}\left|1-\frac{z+n}{z_{i j}}\right| \times \prod_{z_{i j} \in C_{k}}\left|1-\frac{z+n}{z_{i j}}\right| \\
& \quad \times \prod_{z_{i j} \in C_{k+1}}\left|1-\frac{z+n}{z_{i j}}\right| \times\left|\prod_{j=k+2}^{\infty} \prod_{z_{i j} \in C_{j}}\left(1-\frac{z+n}{z_{i j}}\right)\right| \\
> & a_{k} e^{n} \frac{\varepsilon_{0}}{k+1+\left(k^{3}+\frac{k(k+1))}{2}\right) !} \\
& \quad \times \varepsilon_{0}^{2} \frac{\left[\left((k+1)^{3}+2\right) !-\left((k+1)^{3}+1\right) !\right]^{\frac{(k+1)(k+2)}{2}-2}}{\left[k+2+\left((k+1)^{3}+\frac{(k+1)(k+2)}{2}\right) !\right]^{\frac{(k+1)(k+2)}{2}}} \\
> & \varepsilon_{0}^{3} a_{k} e^{n} \frac{\left[\left((k+1)^{3}+2\right) !-\left((k+1)^{3}+1\right) !\right] \frac{(k+1)(k+2)}{2}-2}{\left[k+2+\left((k+1)^{3}+\frac{(k+1)(k+2)}{2}\right) !\right]} .
\end{aligned}
$$

Here we will show that

(3.19)

$$
\log e^{n}-\left(\frac{(k+1)(k+2)}{2}+1\right) \log \left[k+2+\left((k+1)^{3}+\frac{(k+1)(k+2)}{2}\right) !\right]>0 .
$$


Since $n \in N(k), 1+\left(k^{3}+\frac{k(k+1)}{2}\right) !<n$ by the definition of $N(k)$. We apply Stirling's formula to the left of (3.19); then we have

$$
\begin{aligned}
\log e^{n} & -\left(\frac{(k+1)(k+2)}{2}+1\right) \log \left[k+2+\left((k+1)^{3}+\frac{(k+1)(k+2)}{2}\right) !\right] \\
\geq & \left(k^{3}+\frac{k(k+1)}{2}\right) !-\frac{1}{2}(k+2)^{2} \log (k+2)^{3} ! \\
\sim & \left(k^{3}+\frac{k(k+1)}{2}\right) !-\frac{1}{2}(k+2)^{2} \log \sqrt{2 \pi(k+2)^{3}} e^{-(k+2)^{3}}(k+2)^{3(k+2)^{3}} \\
= & \left(k^{3}+\frac{k(k+1)}{2}\right) !+\frac{1}{2}(k+2)^{5} \\
& -\frac{1}{2}(k+2)^{2}\left(3(k+2)^{3} \log (k+2)+\log \sqrt{2 \pi(k+2)^{3}}\right) \\
> & \left(k^{3}+\frac{k(k+1)}{2}\right) !-\frac{3}{2}(k+2)^{5} \log (k+2) \\
> & 0
\end{aligned}
$$

for large enough $k$. This implies

$$
|f(z+n)|>\varepsilon_{0}^{3} a_{k}\left[\left((k+1)^{3}+2\right) !-\left((k+1)^{3}+1\right) !\right]^{\frac{(k+1)(k+2)}{2}-2}
$$

and the right-hand side of (3.20) tends to infinity as $k \rightarrow \infty$. From (3.8), $k \rightarrow \infty$ as $n \rightarrow \infty$, so $|f(z+n)| \rightarrow \infty$ uniformly on the compact set $F$ as $n \rightarrow \infty$. Therefore $z_{0} \in \mathbf{N}(f)$.

3.3. If $z \in G^{c}$, then $z \notin \mathbf{N}(f)$. Since $W$ is a countable subset of $G_{0}^{c}$, for each $w_{s t} \in W$ and $\delta>0$, we can choose a number $\delta_{0}, 0<\delta_{0}<\delta$, so that $w_{s t}+\delta_{0} \neq w_{k l}$ (and $\neq w_{k l}+1$ ) for all $w_{k l} \in W$. From the construction of the sequence $Z, t$ is the smallest integer such that $C_{t}$ contains the translation of $w_{s t}$. And the translations of $w_{s t}$ have the form

$$
z_{\gamma n}=w_{s t}+\left(n^{3}+\gamma\right) !
$$

where $n \geq t$ and $\gamma=\frac{t(t-1)}{2}+s$. We fix $w_{s t}$ and show $\left|f\left(z_{\gamma n}+\delta_{0}\right)\right| \rightarrow \infty$ as $n \rightarrow \infty$.

For each positive integer $n$, we let

$$
\begin{gathered}
I_{n}=\prod_{j=1}^{n-1} \prod_{z_{i j} \in C_{j}}\left(1-\frac{z_{\gamma n}+\delta_{0}}{z_{i j}}\right), \\
J_{n}=\prod_{z_{i j} \in C_{n}}\left(1-\frac{z_{\gamma n}+\delta_{0}}{z_{i j}}\right),
\end{gathered}
$$

and

$$
K_{n}=\prod_{j=n+1}^{\infty} \prod_{z_{i j} \in C_{j}}\left(1-\frac{z_{\gamma n}+\delta_{0}}{z_{i j}}\right)
$$


We fix $n$ for a moment and write

$$
\begin{aligned}
g\left(z_{\gamma n}+\delta_{0}\right) & =\prod_{j=1}^{\infty} \prod_{z_{i j} \in C_{j}}\left(1-\frac{z_{\gamma n}+\delta_{0}}{z_{i j}}\right) \\
& =I_{n} \times J_{n} \times K_{n} .
\end{aligned}
$$

We examine the moduli of $I_{n}, J_{n}$, and $K_{n}$.

(i) Estimation of $\left|I_{n}\right|$ : Assume $\delta_{0}<1$; then by (3.4), for each $z_{i j} \in C_{j}$ where $1 \leq j<n$,

$$
\begin{aligned}
\left|\frac{z_{\gamma n}+\delta_{0}}{z_{i j}}\right| & \geq \frac{n^{3} !}{j+1+\left(j^{3}+\frac{j(j+1)}{2}\right) !} \\
& \geq \frac{n^{3} !}{n+\left((n-1)^{3}+\frac{n(n-1)}{2}\right) !} \\
& >2 .
\end{aligned}
$$

So we obtain

$$
\left|1-\frac{z_{\gamma n}+\delta_{0}}{z_{i j}}\right| \geq|1-| \frac{z_{\gamma n}+\delta_{0}}{z_{i j}}||
$$

and

$$
\left|I_{n}\right|=\prod_{j=1}^{n-1} \prod_{z_{i j} \in C_{j}}\left|1-\frac{z_{\gamma n}+\delta_{0}}{z_{i j}}\right|>1 .
$$

(ii) Estimation of $\left|J_{n}\right|$ : Since

$$
\begin{aligned}
\min & \left\{\left|z_{\gamma n}-z_{i n}\right|: z_{i n} \in C_{n}, z_{i n} \neq z_{\gamma n}\right\} \\
& >\left|z_{1 n}-z_{2 n}\right| \geq \operatorname{Re}\left(z_{2 n}-z_{1 n}\right) \\
& >\left(n^{3}+2\right) !-\left(\left(n^{3}+1\right) !+1\right) \\
& >\left(n^{3}+1\right) !
\end{aligned}
$$

and

$$
\begin{aligned}
\left|J_{n}\right| & =\prod_{z_{i n} \in C_{n}}\left|1-\frac{z_{\gamma n}+\delta_{0}}{z_{i n}}\right| \\
& =\left|1-\frac{z_{\gamma n}+\delta_{0}}{z_{\gamma n}}\right| \times \prod_{\substack{z_{i n} \in C_{n} \\
z_{i n} \neq z_{\gamma n}}}\left|1-\frac{z_{\gamma n}+\delta_{0}}{z_{i n}}\right| \\
& \geq \frac{\delta_{0}}{\left|z_{\gamma n}\right|} \times \prod_{\substack{z_{i n} \in C_{n} \\
z_{i n} \neq z_{\gamma n}}}\left|\frac{z_{i n}-\left(z_{\gamma n}+\delta_{0}\right)}{z_{i n}}\right|
\end{aligned}
$$

with (3.2) and (3.4), we obtain the following inequality

$$
\left|J_{n}\right|>\delta_{0} \frac{\left(\left(n^{3}+1\right) !\right)^{\frac{n(n+1)}{2}}-1}{\left(n+1+\left(n^{3}+\frac{n(n+1)}{2}\right) !\right)^{\frac{n(n+1)}{2}}} .
$$


(iii) $\left\{\left|K_{n}\right|\right\}$ is an eventually increasing sequence: We consider the ratio (for convenience, we let $\left.z_{\gamma \hat{n}}=z_{\gamma(n+1)}\right)$ :

$$
\begin{aligned}
\frac{k_{n+1}}{K_{n}}= & \prod_{j=}^{\infty} \frac{\prod_{z_{i j}^{\prime} \in C_{j+1}}\left(1-\frac{z_{\gamma \hat{n}}+\delta_{0}}{z_{i j}^{\prime}}\right)}{\prod_{z_{i j}} \in C_{j}\left(1-\frac{z_{\gamma n}+\delta_{0}}{z_{i j}}\right)} \\
= & \prod_{z_{i j} \in C_{n+1}} \frac{z_{i j}}{z_{i j}-\left(z_{\gamma n}+\delta_{0}\right)} \times \frac{\prod_{z_{i j}^{\prime} \in C_{n+2}}\left(1-\frac{z_{\gamma \hat{n}}+\delta_{0}}{z_{i j}^{\prime}}\right)}{\prod_{z_{i j} \in C_{n+2}}\left(1-\frac{z_{\gamma n}+\delta_{0}}{z_{i j}}\right)} \\
& \times \frac{\prod_{z_{i j}^{\prime} \in C_{n+3}}\left(1-\frac{z_{\gamma \hat{n}}+\delta_{0}}{z_{i j}^{\prime}}\right)}{\prod_{z_{i j} \in C_{n+3}}\left(1-\frac{z_{\gamma n}+\delta_{0}}{z_{i j}}\right)} \times \cdots \\
= & \prod_{z_{i j} \in C_{n+1}} \frac{z_{i j}}{z_{i j}-\left(z_{\gamma n}+\delta_{0}\right)} \times \frac{\prod_{z_{i j}^{\prime} \in C_{n+2}}\left(z_{i j}^{\prime}-\left(z_{\gamma \hat{n}}+\delta_{0}\right)\right)}{\prod_{z_{i j} \in C_{n+2}}\left(z_{i j}-\left(z_{\gamma n}+\delta_{0}\right)\right)} \\
= & \times \frac{\prod_{z_{i j}^{\prime} \in C_{n+3}}\left(z_{i j}^{\prime}-\left(z_{\gamma \hat{n}}+\delta_{0}\right)\right)}{\prod_{z_{i j} \in C_{n+3}}\left(z_{i j}-\left(z_{\gamma n}+\delta_{0}\right)\right)} \times \cdots \\
z_{i j} \in C_{n+1} & \frac{z_{i j}}{z_{i j}-\left(z_{\gamma n}+\delta_{0}\right)} \times \prod_{z_{i j}^{\prime} \in C_{n+2}}\left(z_{i j}^{\prime}-\left(z_{\gamma \hat{n}}+\delta_{0}\right)\right) \\
& \times \frac{\prod_{z_{i j}^{\prime} \in C_{n+3}}\left(z_{i j}^{\prime}-\left(z_{\gamma \hat{n}}+\delta_{0}\right)\right)}{\prod_{z_{i j} \in C_{n+2}}\left(z_{i j}=\left(z_{\gamma n}+\delta_{0}\right)\right)} \times \frac{\prod_{z_{i j}^{\prime} \in C_{n+4}}\left(z_{i j}^{\prime}-\left(z_{\gamma \hat{n}}+\delta_{0}\right)\right)}{\prod_{z_{i j} \in C_{n+3}}\left(z_{i j}-\left(z_{\gamma n}+\delta_{0}\right)\right)} \\
& \times \cdots
\end{aligned}
$$

The moduli of the first and second parts of the last equation of (3.26) are greater than 1. And for $z_{i j}^{\prime} \in C_{n+l+1}$ and $z_{i j} \in C_{n+l}, l \geq 2$, we have

$$
\begin{aligned}
& \frac{\left|z_{i j}^{\prime}-\left(z_{\gamma \hat{n}}+\delta_{0}\right)\right|}{\left|z_{i j}-\left(z_{\gamma n}+\delta_{0}\right)\right|}>\frac{\left|z_{i j}^{\prime}\right|-\left|z_{\gamma \hat{n}}+\delta_{0}\right|}{\left|z_{i j}\right|} \\
& \quad>\frac{\left((n+l+1)^{3}+1\right) !-\left[(n+1)+1+\left((n+1)^{3}+\frac{(n+1)(n+2)}{2}\right) !\right]}{n+l+1+\left((n+l)^{3}+\frac{(n+l)(n+l+1)}{2}\right) !} \\
& \quad>\frac{\left((n+l+1)^{3}+1\right) !-(n+2)^{3} !}{\left((n+l)^{3}+(n+l)^{2}\right) !} \\
& \quad>1
\end{aligned}
$$

for sufficiently large $n$. Since $\left\|C_{n+l+1}\right\| \geq\left\|C_{n+l}\right\|$,

$$
\frac{\prod_{z_{i j}^{\prime} \in C_{n+l+1}}\left|z_{i j}^{\prime}-\left(z_{\gamma \hat{n}}+\delta_{0}\right)\right|}{\prod_{z_{i j} \in C_{n+l}}\left|z_{i j}-\left(z_{i j}+\delta_{0}\right)\right|}>1
$$

for all $l \geq 2$. Hence we can conclude that $\left|\frac{K_{n+1}}{K_{n}}\right|>1$ for sufficiently large $n$. 
Now we can estimate the value $\left|f\left(z_{\gamma n}+\delta_{0}\right)\right|$. From (3.23), (3.24) and (3.25), we obtain

$$
\begin{aligned}
\left|f\left(z_{\gamma n}+\delta_{0}\right)\right| & =\left|e^{z_{\gamma n}+\delta_{0}} g\left(z_{\gamma n}+\delta_{0}\right)\right| \\
& =\left|e^{z_{\gamma n}+\delta_{0}}\right| \cdot\left|I_{n}\right| \cdot\left|J_{n}\right| \cdot\left|K_{n}\right| \\
& \geq e^{\left(n^{3}+\gamma\right) !-1} \delta_{0} \frac{\left(\left(n^{3}+1\right) !\right)^{\frac{n(n+1)}{2}}-1}{\left(n+1+\left(n^{3}+\frac{n(n+1)}{2}\right) !\right)^{\frac{n(n+1)}{2}}}\left|K_{n}\right| .
\end{aligned}
$$

Here we let

$$
\alpha_{n}=\frac{e^{\left(n^{3}+\gamma\right) !-1}}{\left(n+1+\left(n^{3}+\frac{n(n+1)}{2}\right) !\right)^{n(n+1)}} .
$$

Then for large enough $n$, by Stirling's formula we get

$$
\begin{aligned}
\log \alpha_{n} \geq & \left(n^{3}+\gamma\right) !-\frac{n(n+1)}{2} \log \left(n^{3}+n^{2}\right) ! \\
\sim & \left(n^{3}+\gamma\right) !-\frac{n(n+1)}{2} \log \sqrt{2 \pi\left(n^{3}+n^{2}\right)} e^{-\left(n^{3}+n^{2}\right)}\left(n^{3}+n^{2}\right)^{n^{3}+n^{2}} \\
= & \left(n^{3}+\gamma\right) !+\frac{n(n+1)}{2}\left(n^{3}+n^{2}\right) \\
& \quad-\frac{n(n+1)}{2}\left[\left(n^{3}+n^{2}\right) \log \left(n^{3}+n^{2}\right)+\log \sqrt{2 \pi\left(n^{3}+n^{2}\right)}\right] \\
> & \left(n^{3}+\gamma\right) !-\frac{n^{3}(n+1)^{2}}{2} \log \left(n^{3}+n^{2}\right) \\
> & 0 .
\end{aligned}
$$

So $\alpha_{n}>1$ and the inequality (3.27) becomes

$$
\left|f\left(z_{\gamma n}+\delta_{0}\right)\right|>\delta_{0} \cdot\left|K_{n}\right| \cdot\left(\left(n^{3}+1\right) !\right)^{\frac{n(n+1)}{2}-1} .
$$

Since $\left\{\left|K_{n}\right|\right\}$ is an increasing sequence, $\left|f\left(z_{\gamma n}+\delta_{0}\right)\right| \rightarrow \infty$ as $n \rightarrow \infty$.

But for each positive integer $n$,

$$
\begin{aligned}
f\left(z_{\gamma n}\right) & =f\left(w_{s t}+\left(n^{3}+\gamma\right) !\right) \\
& =0
\end{aligned}
$$

by the definition of the function $f$. So $w_{s t}$ does not belong to $\mathbf{N}(f)$. We choose $w_{s t}$ arbitrarily from the set $W$ and $W$ is a dense subset of $G_{0}^{c}$. Hence no point in $G_{0}^{c}$ can be in $\mathbf{N}(f)$.

\section{REFERENCES}

1. G. D. Birkhoff, Démostration d'un théoréme élémentaire sur les fonctions entières, C. R. Acad. Sci. Paris 189 (1929), 473-475.

2. C. Blair and L. A. Rubel, A universal entire function, Amer. Math. Monthly 90 (1983), 331332. MR 85a:30046

3. J. Clunie and W. K. Hayman, The spherical derivative of integral and meromorphic functions, Comment. Math. Helv. 40 (1966), 117-148. MR 33:282

4. W. K. Hayman, Meromorphic functions, Clarendon Press, Oxford, 1964. MR 29:1337

5. D. H. Luecking and L. A. Rubel, Complex analysis, Springer-Verlag, New York, 1984. MR 86d:30002 
6. F. Marty, Recherches sur la répartition des valeurs d'une fonction méromorphe, Ann. Fac. Sci. Univ. Toulouse (3) 23 (1931), 183-261.

7. Paul Montel, Leçons sur les familles normals de fonctions analytiques et leurs applications, Paris, 1927.

Korea Military Academy, Seoul 139-799, Korea

E-mail address: jkim@hwarang.kma.ac.kr

Department of Mathematics, University of Illinois, Urbana, Illinois 61801 